\title{
M2M Communication
}

\author{
H. Malleck OVE, C. Mecklenbräuker
}

Online publiziert am 18. Jänner 2014

(C) Springer Verlag Wien 2013

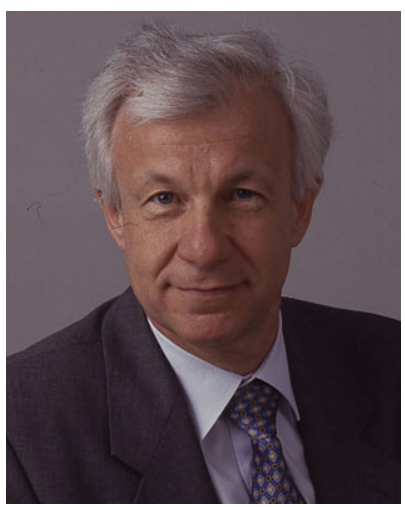

Dipl.-Ing. Dr. Helmut Malleck

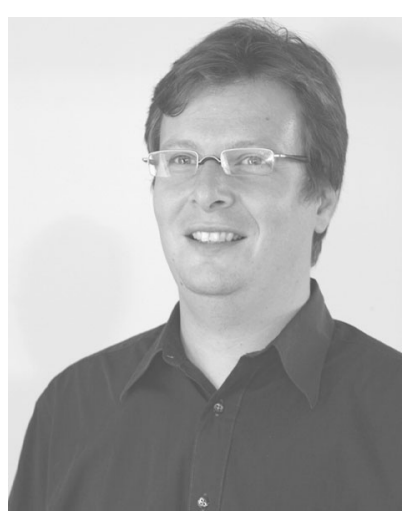

Univ.-Prof. Dipl.-Ing. Dr.-Ing. Christoph Mecklenbräuker

\author{
Liebe Leserinnen und liebe \\ Leser,
}

wir wollten wieder ein aktuelles Informationstechnisches Kolloquium gestalten. Wie schon so oft trafen wir uns dazu im Wiener Café Schwarzenberg. Das Gespräch verlief sachlich, inspiriert aus unseren Tagesgeschäften und von Beiträgen internationaler Fachzeitschriften. Auch gut gemeinte Vorschläge von Kollegen aus Wirtschaft und Forschung standen zur Diskussion.

Machine-to-Machine Communication, das wäre doch etwas! Vom Zuschnitt her allgemein genug, um einer Vielzahl laufender Aktivitäten einen Rahmen zu geben. Das Thema könnte bei österreichischen Ingenieuren und Unternehmern auf Interesse stoßen, setzt doch eine erfolgreiche Umsetzung von Machine-to-MachineLösungen eine enge Kooperation zwischen Lösungspartner, Netzbetreiber und Kunden voraus.

Für 2020 wird vorhergesagt, dass mindestens 12 Milliarden Geräte im Internet verbunden sein werden. Ähnlich wie Peer-to-Peer Communication im Internet, bereits 2006 beim Nachrichtentechnischen Kolloquium machte Henry Sinnreich diesen (US)-Trend deutlich, könnte mit Machine-toMachine Communication eine neue Ära eingeläutet werden. Wir wollten mehr darüber wissen.

Die Anzahl der eingereichten und in diesem M2M-Themenheft veröffentlichten Originalarbeiten und die Beiträge in der Rubrik Praxis \& Wissen machen deutlich, dass sich mit Machine-to-Machine Communication neue Geschäftsmöglichkeiten und spannende Forschungsthemen eröffnen. Wie bereits Tradition, werden ausgewählte Vorträge des aktuell stattgefundenen Informationstechnischen Kolloquiums mit weiteren Beiträgen zu diesem Themenschwerpunkt in diesem e\&i-Heft veröffentlicht.

Einleitend stellt Tim Jones im Leitartikel "The Emerging Role of Mobile: A Look to 2020" die Positionierung von M2M durch strukturiertes Herangehen an die informations- und kommunikationstechnische Zukunft eindrucksvoll dar. Fünf herausragend abgefasste Originalarbeiten, welche die Breite des Themas widerspiegeln, schlieBen daran an.
1. Zunächst zeigen Tanja Zseby, Joachim Fabini und Dipika Rani mit "Synchrophasor Communication" den Einfluss und die praktische Verwendbarkeit von VDSL, HSPA und LTE für M2MKommunikation im Hinblick auf spezielles Monitoring und Steuerung von Smart Grids. Für jedes der ausgewählten Übertragungsprinzipien werden die erzielbaren Durchsatzverzögerungen vergleichend diskutiert.

2. Jesper Groenbaek und Sandford Bessler stellen mit "Impact and control of network QoS on smart grid controlled electrical vehicle charging" ein neues Konzept vor zur Ladestromsteuerung für ein Ensemble von Elektroautos in Verbindung mit der Qualität der elektrischen Niederspannungsnetze und der Datenverzögerung und -sicherheit bei der Übertragung von Smart Meter-Messdaten über Power Line Control und WLAN.

3. Claudia Fösleitner et al. zeigen mit dem Beitrag „M2M-Kommunikation und Satellitennavigation" praktische Anwendungen von Location-based Services mit einem Korrekturdatendienst zur Erhöhung der Positionierungsgenauigkeit in Kombination mit leistungsfähigen Kommunikationsendgeräten. Vorgestellt werden ein mobiles Sicherheitssystem, das die Sicherheit von Personen, die auf abgelegenen Betriebsstätten unterwegs sind, unterstützt und Lösungen, die es durch akustische und haptische Anweisungen blinden und sehbehinderten Menschen ermöglichen, sich selbstständig im öffentlichen Raum zu bewegen.

4. Andreas Hochgatterer et al. geben mit "Ambient Assisted Living - Intelligente Assistenz durch M2M-Technologien" einen guten Überblick über aktuelle österreichische Aktivitäten zu IKTbasierten M2M-Technologieen, die das Leben älterer Menschen situationsabhängig und unaufdringlich unterstützen. Die anwendungsorientieren Forschungsprojekte Gesundheitsdialog Diabetes, iStoppFalls, Füllstandsmessung mittels Near Field Communication und Leichter Wohnen (moduLAAr) werden erörtert.

5. Markus Tauber, Christian Wagner und Frank Pallas stellen mit "Sicherheit und rechtliche Herausforderungen in Bezug auf Cloud Computing und Kritische Infrastruktur-IT" ein technischjuristisches Positionspapier über den Support von Cloud-Computing als M2M-Anwendung für kritische Infrastrukturen bereit. Nach der Abgrenzung zur bestehenden Literatur werden technische Problemstellungen anhand ausgewählter Beispiele angeführt.

Weiterführende Themen zur M2M-Communication werden von drei Beiträgen in der Rubrik Praxis \& Wissen aufgezeigt:

Informationstechnische, zu Beginn Nachrichtentechnische Kolloquien genannt, sind seit 2005 jährlich stattfindende Vortragsveranstaltungen mit aktuellen Themen aus der IT-Welt, hochkarätigen Referenten aus Wirtschaft und Wissenschaft, organisiert von TU Wien, ftw und OVE.

Malleck, Helmut, Dipl.-Ing. Dr., Arbeitskreis für technische Koordination für öffentliche Kommunikationsnetze und -dienste (AK-TK), Postfach 147, 1031 Wien, Österreich (E-Mail: helmut.malleck@oefeg.at); Mecklenbräuker, Christoph, Univ.-Prof. Dipl.-Ing. Dr.-Ing., Institute of Telecommunications, Technische Universität Wien, Gußhausstraße 25 , 1040 Wien, Österreich 
- Wolfgang Leindecker gibt in seinem Beitrag „M2M - Der Reality Check. Wo liegen die Herausforderungen in der Realisierung von M2M-Lösungen?" eine Positionierung von M2M in der aktuellen technischen Landschaft mit deutlich verringerten technischen Einstiegshürden. Aus Kundensicht bringt M2M Optimierungen zur Energieeffizienz und Einsparungen bei Prozessabläufen.

- „Best Practice bei mobilen Datenverbindungen - sicher und einfach gemacht": Wolfgang Schwabl und Christian Kantner, Telekom Austria Group, betrachten zukunftssichere Datenverbindungen für M2M. Mobilfunk-Services, die den Betrieb einer großen Anzahl von M2M-Geräten mit minimierten Risken ermöglichen, werden aufgezeigt.

- „Web of Things und M2M für Konsumenten": Werner WeihsSedivy und Dietmar Millinger, twingz development $\mathrm{GmbH}$, stellen einen innovativen M2M-Lösungsansatz für Zusammenfassungen mit offenen Apps und Ding-Ontologien dar, um eine einfache Integration in Produkte nutzerspezifisch zu ermöglichen.
Wir hoffen, dass Ihnen die hier vorgestellten vielfältigen Beiträge die Möglichkeiten und Chancen von M2M für uns alle deutlich machen können und wünschen Ihnen viel Vergnügen bei der Lektüre der Beiträge dieses Themenheftes.

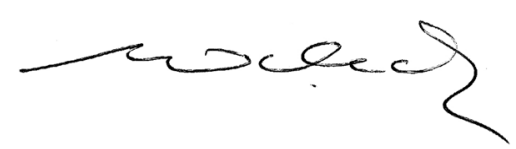

\section{Helmut Malleck}

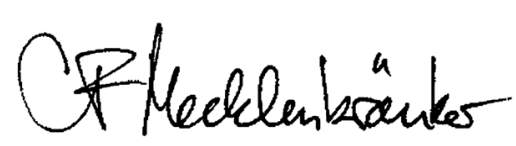

Christoph Mecklenbräuker 